\title{
Method of Algorithms for Cyber-Physical Production Systems Functioning Synthesis
}

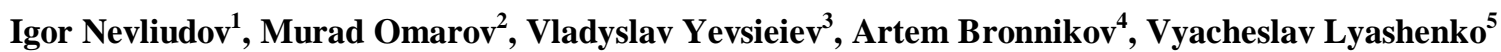 \\ ${ }^{1}$ Department of Computer-Integrated Technologies, Automation and Mechatronics, Kharkiv National University \\ of RadioElectronics, Ukraine, igor.nevliudov@nure.ua \\ ${ }^{2}$ Vice-Rector on International Cooperation, Kharkiv National University of RadioElectronics, Ukraine, \\ murad.omarov@nure.ua \\ ${ }^{3}$ Department of Computer-Integrated Technologies, Automation and Mechatronics, Kharkiv National University \\ of RadioElectronics, Ukraine, vladyslav.yevsieiev@nure.ua \\ ${ }^{4}$ Department of Computer-Integrated Technologies, Automation and Mechatronics, Kharkiv National University \\ of RadioElectronics, Ukraine, artem.bronnikov@nure.ua \\ ${ }^{5}$ Open Digital Group LVV, Kharkiv, Ukraine, lyashenko.vyacheslav@ gmail.com
}

\begin{abstract}
The management process of the new cyber-physical systems (CPPS) development is a complex task that requires the combination of physical and cybernetic components. Now there are no architectural solutions and approaches that allow to automate the management process of CPPS development based on the "end-to-end development" concepts, which will reduce the time and development cost. This article proposes a method for functioning algorithms synthesis. As a result of manipulations, the developer can get many options for algorithms synthesis into one, and the most optimal, according to the requirements that need to be achieved. This method can be implemented as a software in the form of an automation system for the CPPS development control process.
\end{abstract}

Key words: Industry 4.0, Cyber-Physical Production Systems, Digital Twins, Functioning Algorithms.

\section{INTRODUCTION}

Modern high-tech manufacturing imposes new requirements, whose implementation is not possible without the cyber-physical production systems (CPPS) use within the framework of Industry 4.0 concepts [1]-[4]. The development of a production system that takes into account all the factors of interaction between the physical and cybernetic CPPS components based on the concepts of "Digital Twins" will allow to fully automate the production process, which will ensure the Lean Production (LP) goals achievement [5]-[8].
Publications analysis showed that at the moment the proposed the CPPS architecture reference models, such as ISO-95, 5C, $8 \mathrm{C}$ and RAMI 4.0, do not have a single mathematical apparatus, but represent a set of general recommendations, which does not make it possible to automate the process of their development management [9]-[14].

In the publication [15], an architectural and logical model of the complex CPPS development management process automation was proposed, which, in contrast to the existing ones, is a "rigidly" hierarchical structure of logically interrelated mathematical concepts that makes it possible to implement an approach to automate the end-to-end CPPS development management process. During the experiments, it was revealed that this model and methods make it possible to obtain an algorithm for the functioning of each decomposition level, and not a general algorithm for the CPPS functioning, as a consequence of this, in order to solve this problem, it is necessary to develop a new synthesis method that will allow to combine all decomposition levels into a common algorithm for the CPPS functioning, which will automate its development management process.

\section{THE DEVELOPMENT OF CPPS OPERATING ALGORITHMS GROUPING AND SYNTHESIS METHOD}

\subsection{CPPS development method grouping}

The proposed architectural and logical model of the complex CPPS development management process automation analysis [8] showed some patterns: the possibility of using one method or another at different CPPS development levels and stages. As a result, it became necessary to develop a method for CPPS 
Igor Nevliudov et al., International Journal of Emerging Trends in Engineering Research, 8(10), October 2020, 7465 - 7473

functioning algorithms synthesis. In order to achieve this, it is necessary to minimize the number of executed operators, provided that the synthesized algorithm will meet all the conditions of the technical task completely. The proposed method will allow to optimize the CPPS functioning algorithm and achieve economic effect solving problems of management processes for the complex cyber-physical systems development automation.

To do this, it is proposed to group the proposed methods as follows:

- group the goal decomposition methods $\left(\operatorname{Aim}_{i}\right)$, tasks $\left(\right.$ Task $\left._{j}\right)$, structures, algorithms of functioning $(A F)$ top level to bottom level elements;

- group the methods: by operators ( $\left.O p_{h_{-}} A F\right)$, by tasks Task $_{j}$ and object structures (InputCanal, OutCanal ).

Applying the method of transformation can be seen following pattern: goals are transformed to tasks into the task structure, the structure into a communication channel, communication into the object operation algorithm;

- group the methods for calculating the tactical and technical characteristics ( PCofI ) Aim , Task $_{j}$,structures,

InputCanal, OutCanal, $\mathrm{Op}_{h}$;

- group the methods of a system model, target, functional, infological, informational and functioning algorithm development;

- group analytical methods of design solutions strictly according to design stages.

Each CPPS and its constituent parts differ in properties, but during the study, the presence of repetitions was highlighted, therefore it is possible to assert the existence of isomorphisms:

$$
\operatorname{Aim}_{i} \cong \operatorname{Task}_{j} \cong \operatorname{StrE}_{q} \cong I C_{v} \cong O p_{h}
$$

This makes it possible to highlight elements, groups, subsystems and refer them to objects of system design.

\subsection{Method for CPPS structural system models representing}

Let introduce the following definitions: the system model is a graphical representation of the CPPS, where objects corresponding to the stages and levels of design can be represented as nodes, and edges as channels of connections between them.

Based on the proposed decomposition method [8], the representation of system models can be written in the following form:

$$
\operatorname{Aim}_{i_{-}} M S_{0}^{\prime}=\Omega \operatorname{Aim}_{i_{-}} M S_{0}^{\prime \prime}
$$

where: $\operatorname{Aim}_{i_{-}} M S_{0}^{\prime}$ - the main goal of CPPS design in accordance with the technical specifications; $\operatorname{Aim}_{i}{ }_{-} M S_{0}^{\prime \prime}-$ goals at the level of decomposition $M S_{0}^{\prime \prime}$.

Based on 1, you can imagine the decomposition $\mathrm{Aim}_{i-} M S_{0}^{\prime \prime}$ on sub-goals $\mathrm{Sub}_{-} S_{k_{-}} M S_{0}^{\prime \prime}$ :

$$
\operatorname{Aim}_{i_{-}} M S_{0}^{\prime \prime}=\Omega A i m_{i}-S u b_{-} S_{k}-M S_{0}^{\prime \prime}
$$

where $\mathrm{Aim}_{i_{-}} \mathrm{Sub}{ }_{-} S_{k_{-}} M S_{0}^{\prime \prime}-$ goals at the decomposition level $S u b_{-} S_{k}-M S_{0}^{\prime \prime}$.

Thus, the goals of decomposition identities development are constructed throughout the tree:

$$
\mathrm{Aim}_{i_{-}} S u b_{-} S_{k_{-}} M S_{0}^{\prime \prime}=\Omega \operatorname{Aim}_{i_{-}} G\{A E o f S\}_{j}-M S_{0}^{\prime \prime},(3)
$$

where $\operatorname{Aim}_{i_{-}} G\{A E o f S\}_{j}{ }_{-} M S_{0}^{\prime \prime}$ - goal for a group of atomic elements $G\{A E o f S\}_{j-} M S_{0}^{\prime \prime}$.

Similarly (2-3), breaking the goals of an atomic element can be written as:

$$
\begin{aligned}
& \operatorname{Aim}_{i_{-}} G\{A E o f S\}_{j}-M S_{0}^{\prime \prime}= \\
& =\Omega \operatorname{Aim}_{i_{-}}{ }^{A E o f S} S_{i_{-}} M S_{0}^{\prime \prime}
\end{aligned}
$$

where: $\operatorname{Aim}_{i_{-}} A E o f S_{i_{-}} M S_{0}^{\prime \prime}$ - goal of an atomic element $\operatorname{AEofS}_{i}$;

From 1-4, it can be seen that the main goal of CPPS design is a consequence of achieving goals at each decomposition level at each stage of development. Therefore, it can be argued about the existence of "inheritance" $(\rightarrow$ ) goals by the principle of decomposition "top-down" (5).

$$
\begin{aligned}
& \operatorname{Aim}_{i_{-}} M S_{0}^{\prime} \rightarrow \text { Aim }_{i_{-}} M S_{0}^{\prime \prime} \rightarrow \\
& \operatorname{Aim}_{i_{-}} \text {Sub_ } S_{k} M S_{0}^{\prime \prime} \rightarrow \\
& \rightarrow \text { Aim }_{i_{-}} G\{A E o f S\}_{j} M S^{\prime \prime} \rightarrow \\
& \operatorname{Aim}_{i_{-}} \text {AEofS }_{i_{-}} M S_{0}^{\prime \prime}
\end{aligned}
$$

Based on expression 5, it is possible to construct a graph for achieving the main goal of CPPS design, taking into account its multilevelness (Figure 1), which allows to take into account the impact of each other goals achievement within one level of decomposition.

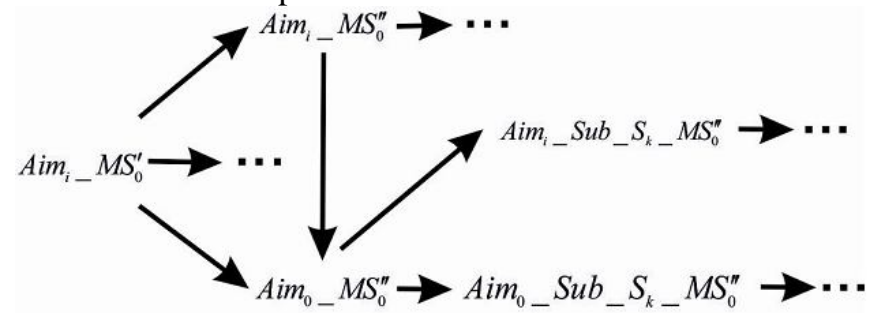

Figure 1: The CPPS design main goal achievement graph. 
Igor Nevliudov et al., International Journal of Emerging Trends in Engineering Research, 8(10), October 2020, 7465 - 7473

Similarly, you can build a graph of the functional level. In this case:

$$
\operatorname{Aim}_{i-} M S_{0}^{\prime}=\Omega \operatorname{Task}_{j-} M S_{0}^{\prime \prime}
$$

where $\operatorname{Aim}_{i_{-}} M S_{0}^{\prime}$ - the main goal of CPPS design; Task $_{j-} M S_{0}^{\prime \prime}$ - tasks of $M S_{0}^{\prime \prime}$ level to reach $\mathrm{Aim}_{i_{-}} M S_{0}^{\prime}$.

Define the nodes of the graph as Task at each level of decomposition, and by the edges of the graph the connection between tasks to achieve the main Task ${ }_{j}$ at this level. This makes it possible to fix connections between tasks using the task counter:

$$
\begin{aligned}
& \text { Task }_{j} \rightarrow \text { Task }_{j+1} \text { Task }_{j} \rightarrow \text { Task }_{j+2} ; \\
& \text { Task }_{j+1} \rightarrow \text { Task }_{j+3} ; \text { Task }_{j+n-1} \rightarrow \text { Task }_{j+n}
\end{aligned}
$$

The proposed "heritage" allows to get a subgraph $\operatorname{Task}_{j}$ representation model, an example of which is shown in Figure 2.

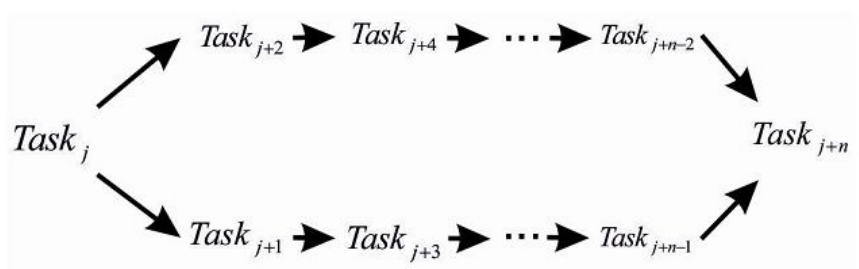

Figure 2: Subgraph Task $j$ representation model.

Based on the subgraph Task ${ }_{j}$ representation model, similarly, we can decompose the next sublevel $S u b_{-} S_{k}-M S_{0}^{\prime \prime}$. Then, for this sublevel, the record is identical to:

$$
\text { Task }_{j}{ }_{-} M S_{0}^{\prime \prime}=\Omega \text { Task }_{j-} \text { Sub }_{-} S_{k_{-}} M S_{0}^{\prime \prime}
$$

where: $T a s k_{j} S_{-} S u b_{-} S_{k} M S_{0}^{\prime \prime}-$ are the tasks at the functional stage of the level $S u b_{-} S_{k}$.

Substituting the subgraph (Figure 2) into the mathematical representation 8, you can get the decomposition graph (Figure 3.) of the main $M S_{0}^{\prime}$ level problem to $S u b_{-} S_{k}$ level.

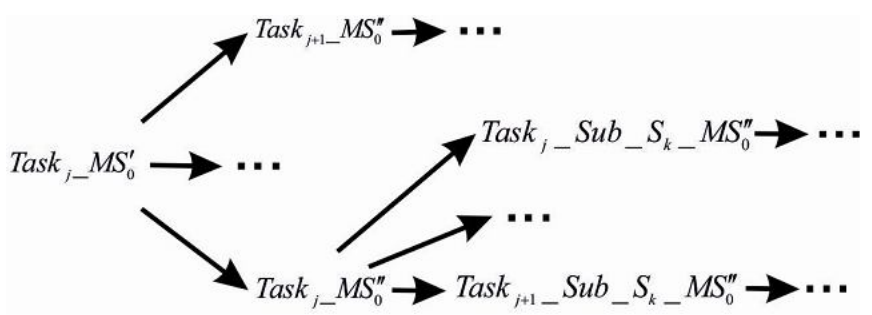

Figure 3: Functional stage graph decompositions to $S u b_{-} S_{k}$ level.

Similarly to expressions 6 and 8, can derive expressions for all levels of decomposition at the functional stage:

$$
\begin{aligned}
& \operatorname{StrE}_{q} \rightarrow \operatorname{StrE}_{q+1} ; \operatorname{StrE}_{q} \rightarrow \operatorname{StrE}_{q+2} ; \\
& \operatorname{StrE}_{q+1} \rightarrow \operatorname{StrE}_{q+3} ; \operatorname{Str} E_{q+n-1} \rightarrow \operatorname{Srt} E_{q+n}
\end{aligned}
$$

To describe the system infological model, let us take the form of a graph node $I C_{v}$ [8], and the edges of the graph will be InputCanal and OutCanal of connections. Based on the developed methodology for constructing a system model $\operatorname{Aim}_{i}$, we describe the systemic infological model of the level $M S_{0}^{\prime \prime}$ :

$$
\begin{aligned}
& I^{M S}{ }_{-} M S_{0}^{\prime}=\Omega I C_{v}-M S_{0}^{\prime \prime}, \\
& \text { InputCanal_IC} C_{v} M S_{0}^{\prime \prime}, \text { OutCanal_IC } C_{v} M S_{0}^{\prime \prime}
\end{aligned}
$$

where: $I^{M S}{ }^{M S} S_{0}^{\prime}$ - infological model of the $M S_{0}^{\prime \prime}$ system level; $I C_{v_{-}} M S_{0}^{\prime \prime}$ - information transformer at the $M S_{0}^{\prime \prime}$ level; InputCanal_IC $C_{v-} M S_{0}^{\prime \prime}$ - input channels at the $M S_{0}^{\prime \prime}$ level; OutCanal_$I C_{v-} M S_{0}^{\prime \prime}$ - output channels at the $M S_{0}^{\prime \prime}$ level.

Similarly to expression 10 can present a systemic model of the infological stage at the following subsystem levels:

- system model of the $I^{M S} \_M S_{0}^{\prime \prime}$ level:

$$
\begin{aligned}
& I^{M S}{ }_{-} M S_{0}^{\prime \prime}=\Omega I C_{v_{-}} S u b_{-} S_{k}, \\
& \text { InputCanal_IC } C_{v} S u b_{-} S_{k}, \\
& \text { OutCanal_IC } C_{v} S_{-} S u b_{-} S_{k}
\end{aligned}
$$

- system model of the $I^{M S}{ }_{-} S u b_{-} S_{k}$ level:

$$
\begin{aligned}
& I^{M S} \_S u b_{-} S_{k}=\Omega I C_{v-} G\{A E o f S\}_{j}, \\
& \text { InputCanal_IC} C_{v-} G\{A E o f S\}_{j}, \\
& \text { OutCanal_IC } C_{v-G}\{A E o f S\}_{j}
\end{aligned}
$$

- system model of the $I^{M S} \_G\{A E o f S\}_{j}$ level:

$$
\begin{aligned}
& I^{M S}{ }_{\text {G }}\left\{\operatorname{AEofS}_{j}=\Omega I C_{v}{ }_{j} A \operatorname{AofS}_{i},\right. \\
& \text { InputCanal_IC }{ }_{v} \text { AEofS } S_{i} \text {, } \\
& \text { OutCanal_IC } C_{v} \text { AEofS } S_{i}
\end{aligned}
$$

- system model of the $I^{M S} \_A E o f S_{i}$ level:

$$
\begin{aligned}
& I^{M S} \_ \text {AEof } S_{i}= \\
& =\Omega \text { EOfS }_{i}\left(\text { InputIP }_{p \_} \_ \text {AEof } S_{i}, \text { OutIP }_{j} \_A E o f S_{i}\right),
\end{aligned}
$$

Based on the infological stage design results (11-14), the developer can begin to implement the system models of the CPPS development information stage - system tactical and technical characteristics of the physical information model ( PCofI_PIM ) at all levels of CPPS decomposition. Based on the proposed methods, it is possible to present a system model of the information stage at the following subsystem levels:

- system model of the PCofI_PIM _ MS $S_{0}^{\prime \prime}$ level: 
Igor Nevliudov et al., International Journal of Emerging Trends in Engineering Research, 8(10), October 2020, 7465 - 7473

$$
\begin{aligned}
& \text { PCofI_PIM_MS } S_{0}^{\prime \prime}= \\
& =\Omega I^{M S} \_S u b_{-} S_{k}-M S_{0}^{\prime \prime}, P C o f I_{-} P I M_{-} S_{u} b_{-} S_{k}
\end{aligned}
$$

- system model of the $P C o f I_{-} P I M_{-} S u b_{-} S_{k}$ level:

$$
\begin{aligned}
& P C o f I_{-} P I M M_{-} S u b_{-} S_{k}=\Omega I^{M S}{ }_{-} G\{A E o f E\}_{j}-M S_{0}^{\prime \prime}, \\
& P C o f I_{-} P I M_{-} G\{A E o f E\}_{j}
\end{aligned}
$$

- system model of the PCofI_PIM_G\{AEofE $\}_{j}$ level:

$$
\begin{aligned}
& P C o f I_{-} P I M_{-} G\{A E o f E\}_{j}= \\
& =\Omega I^{M S}{ }_{-} A E o f S_{i_{-}} M S_{0}^{\prime \prime}, P C o f I_{-} P I M_{-} A E o f S_{i}
\end{aligned}
$$

- system model of the PCofI_PIM_AEofS $S_{i}$ level:

$$
\begin{aligned}
& \text { PCofI_PIM_AEofS } I_{i}= \\
& =\Omega P C o f I_{-} I n p u t I P_{p}-A E o f S_{i}, \\
& \text { PCofI_OutIP }{ }_{p}{ }_{2} A E o f S_{i}
\end{aligned}
$$

To describe the system information model, we take the form of this level node PCofI_PIM, and the edges will be the tactical and technical characteristics of information links ( PCofI_InputIP $P_{p}$, PCofI_OutIP $\left.P_{p}\right)$. The developer is obliged to comply with the condition for the existence of binary relations PCofI_OutIP $p_{p}$ AEofS $_{o}$ and PCofI_InputIP $P_{p \_}$AEofS : $_{i}$

$$
P C o f I_{-} P I M_{2} A E o f S_{0} \cong P C o f I_{-} P I M_{-} \text {AEof } S_{i}
$$

The last stage of CPPS design in accordance with the proposed system design and control representation in the complex CPPS development is the functioning algorithm construction $(A F)$ in this study is proposed to use the principle of graph-schemes due to their convenience of presentation. Therefore, the following method is proposed for their construction:

- the developer analyzes $\operatorname{Aim}_{i}$, Task $_{j}$, InputCanal, OutCanal of the system-level design;

- according to each Task $_{j}$ choose $O p_{h}$ graph-scheme of the algorithm based on the proposed method [8]. Let's designate that the first task of the $T a s k_{0} M S_{0}{ }^{\prime}$ level, will be $O p_{0}$, therefore, for the next $T a k_{0}-M S_{0}{ }^{\prime}$ level of decomposition will be $T a k_{1}-M S_{0}{ }^{\prime \prime}$ level, and will match the $O p_{1}$ operator and by analogy Task ${ }_{j}-M S_{o}^{\prime \prime}$ operator will be $O p_{h}$;

- analyzing the sequence of $\operatorname{Tas}_{j}{ }_{-} M S_{o}^{\prime \prime}$ execution for a given level, the developer reaches $\operatorname{Aim}_{i_{-}} M S_{0}^{\prime \prime}$, necessary to achieve the main goal of the CPPS design;

- the developer arranges $O p_{h}$ according to the logical sequence to execute Task $_{j-} M S_{o}^{\prime \prime}$. Let introduce the concept of conditional and unconditional transition from Task $_{j-} M S_{o}^{\prime \prime}$ to Task $_{j+1}-M S_{o}^{\prime \prime}$, if transition is without condition then $O p_{h}$ connect to $\rightarrow$, if $O p_{h}$ has connections with other operators $O p_{h+1}$ and by condition, then it is necessary to maintain the concept of a conditional operator $\left(\mathrm{CO}_{l}\right)$, which works by analogy with the block "condition" from the basic theory of the algorithm construction. Based on this assumption $A F$ can take into account not only the "linear view", but also implement the "disjunctive transition", "cycles" and "transition conditions" to achieve Task $_{j}$.

\subsection{System models formalization}

To formalize the system models developed in Section 2.2, in this research it was proposed to use the mathematical apparatus of regular algorithms and algebra of logic. Based on the theory of the regular schemes and algorithmic algebras apparatus, in this study we define the following notation:

$O A_{q}$ - set of the algebra elements operators, are $O p_{h}$, and conduct additional operations for the convenience of presentation and are not operators of the information transformation. $H$-identity operator, $\varnothing$ - empty operator;

$A P_{r}$ - a set of conditions that include all logical conditions $\mathrm{CO}_{l}$, and can take the following values true, false or $\mathrm{CO}_{l}^{x}$ $x=[$ true, false $, b, c, y, \ldots, r]$.

Therefore, it can be represented in the form of a tuple $O A_{q}$ :

$$
O A_{q}=\left(O p_{h}, C O_{l}, H, \varnothing, \text { true }, \text { false }, x\right)
$$

For ease of manipulation $O A_{q}$ (within algorithmic algebras) it is necessary to define the main types of operations:

Definition 1. Operator multiplication - strictly sequential execution of operators in the order of their queue.

$$
\left.O A=O A_{i} \cdot \underset{C O_{l}}{(} O A_{k} \vee O A_{n}\right) \cdot O A_{m}
$$

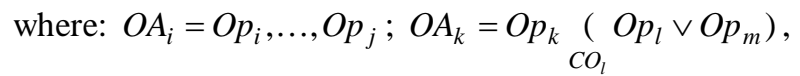
where $C O_{l}$ - logical conditions; $O A_{n}=O p_{n}\left\{O p_{p}\right\} O p_{t}$; $O A_{m}=O p_{m}, \ldots, O p_{s}$.

Definition 2. Operators Addition - is a conditional simple operation branching ( $\left.\tilde{O} p_{h}\right)$ or nested within each other.

$$
O A_{q}=\tilde{O} p_{1} \cdot \tilde{O} p_{2} \cdot, \ldots, \tilde{O} p_{h}
$$


Igor Nevliudov et al., International Journal of Emerging Trends in Engineering Research, 8(10), October 2020, 7465 - 7473

$$
\begin{aligned}
& O A_{q-1}=\underset{C O_{1}}{\left(\tilde{O} p_{1} \vee \underset{C O_{2}}{\left(\tilde{O} p_{2} \vee\right.}{\underset{C O}{C O}}_{3} \tilde{O} p_{3} \vee \ldots\right.} \\
& \ldots \vee \underset{C O_{1}}{\left.\left.\left(\tilde{O} p_{h} \vee e\right)\right)\right)}
\end{aligned}
$$

Using expression 23 as an example, this study suggests the following understanding: ( $\left.\tilde{O} p_{h} \vee e\right)$ the results of addition of simple operations must satisfy the $\mathrm{CO}_{l}$ condition, after that, addition is performed to the next $\mathrm{CO}_{3}$ condition, etc.

Definition 3. The addition process in the system model is the observance of conditional branching and connection of the algorithm paths forward depending on $\mathrm{CO}_{l}$. The syntax for writing is represented as expression 24 :

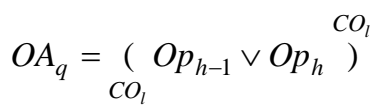

An example of representing the addition process in the system model can be represented by the following expression:

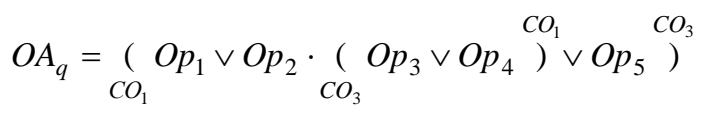

Based on studies by the expression 25 system models can be described iteration "forward" after checking conditions $\mathrm{CO}_{l}$, in this case, it is necessary to characterize the frequently occurring "back" iteration, in which the return is carried out by $O p_{h}$, not to a condition $C O_{l}$. This requires a new definition in the developed formalization.

Definition 4. The iterative addition process is the rules for writing and reading sequential and concentrically nested loops. Based on this definition, we represent the existing types of loops:

- simple sequence of loops

$$
C Y=C Y_{1} \cdot C Y_{2} \cdot \ldots C Y_{n}
$$

where: $\mathrm{CY}_{\mathrm{n}}=\left\{O p_{h}\right\}$;

- concentric of nested loops:

$$
C Y_{n}=\left\{\left\{\left\{\ldots\left\{O p_{1}\right\} \mathrm{CO}_{1} \mathrm{Op}_{2} \mathrm{CO}_{2} \mathrm{Op}_{3} \mathrm{CO}_{3} \ldots O p_{h}\right\}\right.\right.
$$

- a special case of a loop $C Y_{n}$ with return to operator $O p_{h}$ when the condition $\mathrm{CO}_{l}$ performed:

$$
C Y_{n}=\left\{O p_{h-2} \ldots O p_{h-1}\right\} O O_{l} O p_{h}
$$

Based on definitions 1-4, the developer can provide the following description of the designed algorithm iterative paths:

- execution of an interactive way by the condition $C O_{l-1}=$ false, until the closing curly brace and repeat, then return to the check if $\mathrm{CO}_{l}=$ true, then the action of the algorithm is carried over the closing brace to the next operator.

$$
C Y_{n-3}=\underset{C O_{l-1}}{\left\{O p_{1} \ldots O p_{h-1}{ }^{C O_{l}} O p_{h}\right.}
$$

- execution of an interactive path conditionally $C O_{l-1}=$ false, return to open brace and repeat. By condition $\mathrm{CO}_{l}=$ true the action wraps behind the closing brace.

$$
\left.C Y_{n-m}=\stackrel{C O_{l-1}}{O^{\prime}} O p_{1} \ldots O p_{h-1}\right\} O \mathrm{CO}_{h}
$$

Condition $\mathrm{CO}_{l}$ presented below the braces, determines the check of it depending on its parameter (false,true) and depending on the given parameter, make a conditional jump. Condition $\mathrm{CO}_{l-1}$ presented above the brace indicate where to return when the parameter false and continue the algorithm. An example of this type of the algorithm description is presented in 31 .

$$
\begin{aligned}
& O A_{q}=\stackrel{C O}{l-1}^{C O p_{h-5}} \underset{C O_{l-3}}{\left(\left(O p_{h-4} \vee O p_{h-3}\right.\right.} \stackrel{C O_{l-3}}{)} \\
& { }^{C O_{l}} \underset{C O_{l-2}}{(} O p_{h-2} \underset{C O_{l-1}}{(} \vee O p_{h-1} \stackrel{C O_{l-2}}{)} \underset{C O_{l}}{O} O p_{h}
\end{aligned}
$$

Definition 5: The conjunction of the algorithm is an unconditional branching into the execution of several parallel paths of the algorithm.

$$
O A_{q}=\left[O p_{h-2} \wedge O p_{H-1} \wedge O p_{h}\right]
$$

In this study, the following assumption was made that the actions of the algorithm operators enclosed in square brace start in parallel after the opening brace. For this record to be correct, all paths must be equal to exp. 33, therefore we define $P$ - path length (number of operators) in this branch of the algorithm.

$$
P O p_{h-2}=P O p_{H-1}=P O p_{h}
$$

When a condition occurs $P O p_{h-2} \neq P O p_{H-1}$ it is necessary to add $n$ number of operators to the shorter length of the algorithm before condition 33 .

To determine the algorithm branches is proposed the following recording method. $\mathrm{CO}_{l}^{x}$ is indicated at the bottom of the opening square bracket, which means checking the 
Igor Nevliudov et al., International Journal of Emerging Trends in Engineering Research, 8(10), October 2020, 7465 - 7473

condition and the beginning of the parallel operation of the algorithm when $x$, at the top above the closing brace is the exit condition $C O_{l-1}^{x}$ from the algorithm. It should be noted that when executing parallel algorithms, it is necessary to take into account the $x$ parameter at $\mathrm{CO}_{l}$ must be determined by the dimension of conditions and must always be determined for each specific condition $x=[$ true, false $, b, c, y, \ldots, r]$, fulfillment of conditions $b, c, r$, which satisfies the requirements of the exit conditions $\mathrm{CO}_{l-1}$, we can assume that the parallel algorithm has fulfilled its function. An example recording is presented in expression 34.

$$
O A_{q}=\underset{C O_{1}^{X}}{[} O p_{h-4} \wedge \stackrel{b}{\wedge} \wedge \stackrel{r}{r} p_{H-1} \wedge O p_{h}{ }^{C O_{l-1}^{x}}
$$

Similarly, the possibility of existence $\mathrm{CO}_{l}^{x}$ in $x=[$ true false $, b, c, y, \ldots, r]$ for the operation of the adding algorithms iterative process. For the convenience of writing the system model, the following syntax rules are proposed:

- check condition $\mathrm{CO}_{l}$ written below the closing curly brace;

- define the following notation as defining superscripts for curly braces $C O_{l}^{\text {true }, \text { false }, b, c, y,{ }^{\circ}, r}$, and place it in the place where the action of the algorithm returns depending on the values condition $x=[$ true false $, b, c, y, \ldots, r]$

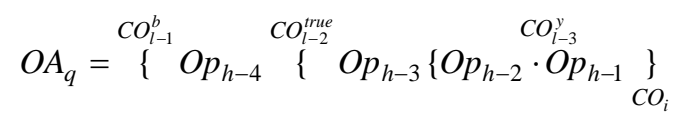

On the basis of the developed system models formalizations language, the developer can carry out an algebraic description of all operators and the conditions of their interaction in the form of an algorithm of functioning ( $A F$ ) at all stages and levels of CPPS decomposition.

Use one space after periods and colons. Hyphenate complex modifiers: "zero-field-cooled magnetization." Avoid dangling participles, such as, "Using (1), the potential was calculated." [It is not clear who or what used (1).] Write instead, "The potential was calculated by using (1)," or "Using (1), we calculated the potential."

\section{METHOD FOR THE CPPS OPERATING ALGORITHMS SYNTHESIS}

System models formalization makes it possible to develop an algorithm for the functioning of a certain stage at the CPPS design chosen level, but at the same time it does not solve the general problem of their interaction as a whole to solve $\operatorname{Aim}_{i_{-}} M S_{0}^{\prime}$. Therefore, the problem arises of synthesizing private algorithm of functioning $A F$ into a general. Based on the above, this study proposed the following synthesis methods:

Definition 6. Functioning algorithms combining - if the set $O p_{h}$ to execute private algorithms is general and $\mathrm{CO}_{l}$ is also common, therefore they can be combined by $\mathrm{CO}_{l}$.

$$
\left.A F=\underset{O C_{l}}{(} A F_{r} \vee A F_{m}\right)
$$

provided that $\mathrm{CO}_{l}=($ true, false $)$

$$
\begin{aligned}
& A F_{r}+A F_{m}=\underset{O C_{l}}{\left(A F_{r} \vee A F_{m}\right)=} \\
& =\left\{\begin{array}{l}
A F_{r} \text { при } C O_{l}=\text { true } \\
A F_{m} \text { при } C O_{l}=\text { false }
\end{array}\right.
\end{aligned}
$$

Based on 36-37, the developer can apply the system of algorithms identical transformations axioms, which will make it possible to simplify the implemented algorithm logical structure.

Definition 7. Decomposition of functioning algorithms is a breakdown of the algorithm into simple algorithms without losing the identities of its operation conditions. An example of the functioning algorithm 38 decomposition is presented in $39-40$.

$$
\begin{aligned}
& A F_{i}=\underset{O C_{l-2}}{\left(A F_{j} \vee \underset{O C_{l-1}}{\left(A F_{m} \vee A F_{n}\right.}\right)^{O C_{l-1} O C_{l-2}} .} . \\
& \cdot \underset{O C_{l}}{\left(A F_{p} \vee A F_{g}\right)}{ }^{O C_{l}}
\end{aligned}
$$

- enlarged example of decomposition:

$$
A F_{i}=\left\{\begin{array}{l}
A F_{1}=A F_{j}\left(A C_{l}\right. \\
\text { when } O C_{l-2}=\text { true } \\
\left.A F_{2}=\underset{O C_{l-1}}{(} A F_{m} \vee A F_{n}\right) \underset{O C_{l}}{(}\left(A F_{p} \vee A F_{g}\right) \\
\text { when } O C_{l-2}=\text { false }
\end{array}\right.
$$

- path is a fragment $A F_{i}$ which contains a specific sequence $O p_{h}$, by the algorithm will be $O p_{h-1} \rightarrow O p_{h}$. Hence it follows that the path of the algorithm is simple, which have no branching, and which is complex, contains: iterative process (definition 4), conjunction (definition 5), etc.

- path lenth - time characteristic of the algorithm and is used to prove the identity of the algorithm for the structural minimization of the same paths.

- detailed example of decomposition: 


$$
A F_{i}=\left\{\begin{array}{l}
A F_{1}=\left\{\begin{array}{l}
A F_{1}^{\prime}=A F_{j} \cdot A F_{p} \\
\text { when }\left(O C_{l-1} \wedge O C_{l}\right)=\text { true } \\
A F_{2}^{\prime \prime}=\left(A F_{m} \vee A F_{n}\right) A F_{g} \\
\text { when }\left(O C_{l-1} \wedge O C_{l}\right)=\text { false }
\end{array}\right. \\
A F_{2}=\left\{\begin{array}{l}
A F_{j}\left(A F_{p} \vee A F_{g}\right) \\
\text { when }\left(O C_{l-2} \wedge O C_{l-1}\right)=\text { true } \\
A F_{n}\left(A F_{p} \vee A F_{g}\right) \\
\text { when }\left(O C_{l-2} \wedge O C_{l-1}\right)=\text { false }
\end{array}\right.
\end{array}\right.
$$

Denote $\vec{A} F_{i}$ as a partial algorithm or algorithm path.

$$
\vec{A} F_{i}=\vec{A} F_{1} \cdot \vec{A} F_{2} \cdot \vec{A} F_{3} \cdot \ldots \cdot \vec{A} F_{i-1}
$$

To combine the original algorithms, it is necessary to decompose it in accordance with Definition 7 and determine simple paths, then analyze $O p_{h}$ and $O C_{l}$ for each $\rightarrow$ from $A F_{i}$ to identify the same.

Axiom 1. Suppose any two algorithms $\vec{A} F_{i}$ and $\vec{A} F_{i-1}$ будут тождественно эквивалентны, will be identically equivalent if they consist of the same $\rightarrow$ path lengths, $O p_{h}$ and $O C_{l}$ in $\rightarrow$ will be equivalent, $O C_{l}$ - is identical.

An example of Axiom 1. Let any three algorithms $42-44$ be given:

$$
\begin{aligned}
& \vec{A} F_{1}=O p_{1} \cdot O p_{2} \underset{O C_{1}}{(O} O p_{3} \vee O p_{4}{ }^{O C_{1}} \\
& \left.\stackrel{O C_{2}}{\{} O p_{5} \cdot O p_{6}\right\} \underset{O C_{2}}{A} \vec{A} F_{i-1} \\
& \vec{A} F_{2}=\underset{O C_{1}}{\left(O p_{3} \vee O p_{4}\right.} \stackrel{O C_{1}}{O} O p_{1} . \\
& \left.\cdot O p_{2} \stackrel{O C_{2}}{\{} O p_{5} \cdot O p_{6}\right\} \overrightarrow{O C} F_{i-1} \\
& \left.\vec{A} F_{3}=\vec{A} F_{i-1} \cdot O p_{1} \cdot O p_{2} \underset{O C_{1}}{\left(O p_{3} \vee O p_{4}\right.}\right)^{O C_{1}} \\
& \left\{C_{2} O p_{5} \cdot O p_{6}\right\}
\end{aligned}
$$

As you can see that the algorithms $\vec{A} F_{1}, \vec{A} F_{2}$ and $\vec{A} F_{3}$ have the same operators $\left(O p_{1}, O p_{2}, O p_{3}, O p_{4}, O p_{5}, O p_{6}\right)$ and conditions $\left(O C_{1}, O C_{2}\right)$, are the same $\rightarrow$. To obtain a combined algorithm and simplify data manipulation, we use the following abbreviations.

$$
O p_{1} \cdot O p_{2}=W
$$

$$
\begin{gathered}
\left.\underset{O C_{1}}{\left(O p_{3} \vee O p_{4}\right.}\right)^{O C_{1}}=M \\
\stackrel{O C_{2}}{\left\{O p_{5} \cdot O p_{6}\right.} \underset{O C_{2}}{ }=K \\
\vec{A} F_{i-1}=Z
\end{gathered}
$$

Substitute the accepted abbreviations from 45-48 to 42-44, and we get the following form of recording the algorithms presented by 49-51:

$$
\begin{aligned}
& \vec{A} F_{1}=W M K Z \\
& \vec{A} F_{2}=M W K Z \\
& \vec{A} F_{3}=Z W M K
\end{aligned}
$$

Let us define additional conditions for the algorithms existence $\vec{A} F_{1}, \vec{A} F_{2}$ и $\vec{A} F_{3}$ using systems:

$$
\begin{aligned}
& \hat{S}_{1}=\left\{\begin{array}{l}
\vec{A} F_{1} \text { when } s_{1}=\text { true } \\
\vec{A} F_{1} \vec{A} F_{2} \text { when } s_{1}=\text { false }
\end{array}\right. \\
& \hat{S}_{2}=\left\{\begin{array}{l}
\vec{A} F_{2} \text { when } s_{2}=\text { true } \\
\vec{A} F_{3} \text { when } s_{2}=\text { false }
\end{array}\right.
\end{aligned}
$$

At the first step of the algorithms synthesis $\vec{A} F_{1}, \vec{A} F_{2}$ and $\vec{A} F_{3}$ the developer needs to determine the same paths according to the criterion of least repeatability and make a record according to the conditions of existence $\vec{A} F_{1}, \vec{A} F_{2}$ and $\vec{A} F_{3}$. As a result of manipulations, the developer can get many options for synthesizing algorithms into one, and depending on the requirements that need to be achieved, the most optimal is selected. Expression 54 gives an example of an algorithm synthesis by the minimizing the number of operators criterion using the introduced abbreviations from 49-51, taking into account additional conditions 52-53.

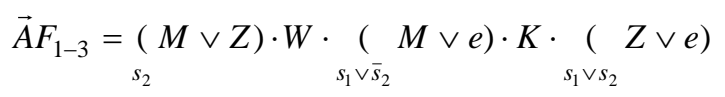

Let us substitute 54 accepted abbreviations into 45-48 and obtain the synthesized algorithm $\vec{A} F_{1-3}$ as a set of operators and a condition.

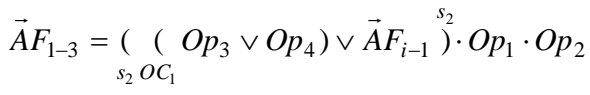

$$
\begin{aligned}
& \left.\left.\underset{s_{1} \vee \bar{s}_{2} O C_{1}}{(} O p_{3} \vee O p_{4}\right) \vee e^{s_{1} \vee \bar{s}_{2}}\right)^{(}\left\{O p_{5} \cdot O p_{6}\right\} \\
& \left.\underset{s_{1} \vee s_{2}}{(} \vec{A} F_{l-1} \vee e\right)
\end{aligned}
$$

By analyzing the synthesis result of the combined algorithm 55 and the original algorithms $42-44$, the developer can see 
Igor Nevliudov et al., International Journal of Emerging Trends in Engineering Research, 8(10), October 2020, 7465 - 7473

that in the algorithms $\vec{A} F_{1}, \vec{A} F_{2}$ and $\vec{A} F_{3}$ there are 21 operators and 6 conditions for a given criterion about the need to minimize operators, 10 operators were obtained while maintaining the number of conditions. The number of operators in this synthesis was reduced by 2 times, while the algorithm fulfills all the conditions set initially. Figure 4 graphically shows a comparison of the execution parameters of the synthesized and non-synthesized algorithm from the example with the number of conditions $=6$.

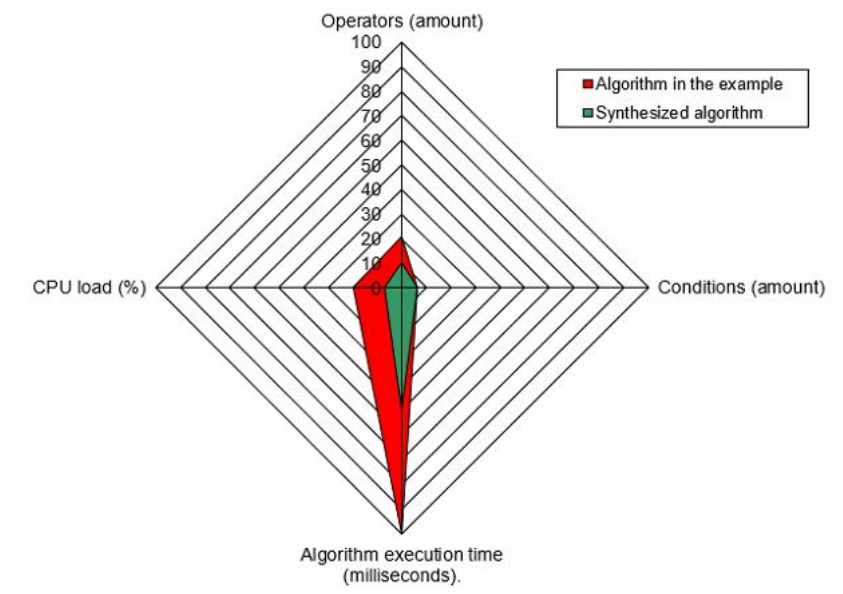

Figure 4: Comparison of the synthesized execution parameters and non-synthesized algorithm.

\section{CONCLUSION}

On the basis of the functioning algorithms synthesis developed method, theoretical studies of the three algorithms synthesis were carried out, which showed: at the initial stage there were 21 operators and 6 conditions under a given constraint conditions - minimization of operators. As a result of the proposed synthesis method approbation 10 operators were obtained while maintaining the number of conditions, which will make it possible to minimize the general CPPS functioning algorithm by 1.5 - 2 times, while the synthesized algorithm fulfills all the initially set conditions. Summing up, it can be argued that the developed synthesis method makes it possible to automate the process of creating a generalized algorithm for the CPPS functioning, which will reduce the development time and achieve the maximum economic effect.

\section{REFERENCES}

1. V.Alcácera, and V. Cruz-Machado. Scanning the Industry 4.0: A Literature Review on Technologies for Manufacturing Systems, Journal of Engineering Science and Technology, Vol. 22, no. 3, pp. 899-919, 2019.

https://doi.org/10.1016/j.jestch.2019.01.006

2. Y. Lu. Industry 4.0, Journal of a Survey on Technologies, Applications and Open Research, Vol. 6, pp. 1-10, 2017. https://doi.org/10.1016/j.jii.2017.04.005
3. P. Leitão, A. W. Colombo, and S. Karnouskos. Industrial automation based on cyber-physical systems technologies: Prototype implementations and challenges, Journal of Computers in Industry, Vol. 81, pp. 11-25, 2016.

https://doi.org/10.1016/j.compind.2015.08.004

4. Y. Liu, Y. Peng, B. Wang, S. Yao, and Z. Liu. Review on cyber-physical systems, IEEE/CAA Journal of Automatica Sinica, Vol. 4, no. 1, pp. 27 - 40, 2016. https://doi.org/10.1109/JAS.2017.7510349

5. T. Wagner, Ch. Herrmann, and S. Thiede. Industry 4.0 Impacts on Lean Production Systems, Journal of Procedia CIRP, Vol. 63, pp. 125-131, 2017. https://doi.org/10.1016/j.procir.2017.02.041

6. M. Schluse, M. Priggemeyer, L. Atorf, and J. Rossmann. Experimentable Digital Twins-Streamlining Simulation-Based Systems Engineering for Industry 4.0, IEEE Transactions on Industrial Informatics, Vol. 14, no. 4, pp. 1722 - 1731, 2018.

https://doi.org/10.1109/TII.2018.2804917

7. Y. Cai, B. Starly, P. Cohen, and Y.-Sh. Lee. Sensor Data and Information Fusion to Construct Digital-twins Virtual Machine Tools for Cyber-physical Manufacturing, Procedia Manufacturing, Vol. 10, pp. 1031-1042, 2017. https://doi.org/10.1016/j.promfg.2017.07.094

8. F. Tao, Q. Qi, L. Wang, and A. Y. C. Nee. Digital Twins and Cyber-Physical Systems toward Smart Manufacturing and Industry 4.0: Correlation and Comparison, Engineering, Vol. 5, no. 4, pp. 653-661, 2019. https://doi.org/10.1016/j.eng.2019.01.014

9. M. A. Pisching, M. A. O. Pessoa, F Junqueira, D. J. S. Filho, and P. E. Miyagi. An architecture based on RAMI 4.0 to discover equipment to process operations required by products, Computers \& Industrial Engineering, Vol. 125, pp. 574-591, 2018. https://doi.org/10.1016/j.cie.2017.12.029

10. O. Kuzomin, M. A. Ahmad, H. Kots, V. Lyashenko, and M. Tkachenko. Preventing of technogenic risks in the functioning of an industrial enterprise, International Journal of Civil Engineering and Technology, Vol. 7, no. 3, pp. 262-270. 2016.

11. E. P. Garina, A.P. Garin, E. V. Romanovskaya, N. S. Andryashina, and $\mathrm{Zh}$. V. Smirnova. The study of approaches for the coordination of product development systems and production systems in the stage of conceptual design, International Journal of Emerging Trends in Engineering Research, Vol. 8, no. 9, pp. 5746-5749, 2020. https://doi.org/10.30534/ijeter/2020/135892020

12. E. Egorova, and A. Akhmadiev. Formation of Engineering Capability Matrix of Production Procedure, International Journal of Emerging Trends in Engineering Research, Vol. 8, no. 9, pp. 6537-6540, 2020.

https://doi.org/10.30534/ijeter/2020/258892020 
13. J.-R. Jiang. An improved cyber-physical systems architecture for Industry 4.0 smart factories, Journal of Advances in Mechanical Engineering, Vol. 10, no. 6, pp. 1-15, 2018.

https://doi.org/10.1177/1687814018784192

14. A. M. Babker, A. E. A. Altoum, I. Tvoroshenko, and V. Lyashenko. Information technologies of the processing of the spaces of the states of a complex biophysical object in the intellectual medical system health, International Journal of Advanced Trends in Computer Science and Engineering, Vol. 8, no 6, pp. 3221-3227.

https://doi.org/10.30534/ijatcse/2019/89862019

15. I. Nevliudov, V. Yevsieiev, S. Maksymova, and I. Filippenko. Development of an architectural-logical model to automate the management of the process of creating complex cyber-physical industrial systems, Eastern-European Journal of Enterprise Technologies, Vol. 4, no. 3 (106), pp.44-52, 2020.

https://doi.org/10.15587/1729-4061. 2020.210761 